\title{
Molecular epidemiology of Plasmodium species prevalent in Yemen based on $18 \mathrm{~s}$ rRNA
}

Abdulsalam MQ Al-Mekhlafi ${ }^{*}$, Mohammed AK Mahdy $^{1,2}$, Ahmed A Azazy $^{2}$, Mun Yik Fong ${ }^{1}$

\begin{abstract}
Background: Malaria is an endemic disease in Yemen and is responsible for 4.9 deaths per 100,000 population per year and 43,000 disability adjusted life years lost. Although malaria in Yemen is caused mainly by Plasmodium falciparum and Plasmodium vivax, there are no sequence data available on the two species. This study was conducted to investigate the distribution of the Plasmodium species based on the molecular detection and to study the molecular phylogeny of these parasites.

Methods: Blood samples from 511 febrile patients were collected and a partial region of the $18 \mathrm{~s}$ ribosomal RNA (18 s rRNA) gene was amplified using nested PCR. From the 86 positive blood samples, 13 Plasmodium falciparum and 4 Plasmodium vivax were selected and underwent cloning and, subsequently, sequencing and the sequences were subjected to phylogenetic analysis using the neighbor-joining and maximum parsimony methods.

Results: Malaria was detected by PCR in 86 samples (16.8\%). The majority of the single infections were caused by P. falciparum (80.3\%), followed by $P$. vivax (5.8\%). Mixed infection rates of $P$. falciparum $+P$. vivax and $P$. falciparum $+P$. malariae were $11.6 \%$ and $2.3 \%$, respectively. All $P$. falciparum isolates were grouped with the strain 3D7, while

P. vivax isolates were grouped with the strain Salvador1. Phylogenetic trees based on $18 \mathrm{~s}$ rRNA placed the

$P$. falciparum isolates into three sub-clusters and P. vivax into one cluster. Sequence alignment analysis showed $5-14.8 \%$ SNP in the partial sequences of the $18 \mathrm{~s}$ rRNA of $P$. falciparum.

Conclusions: Although P. falciparum is predominant, P. vivax, P. malariae and mixed infections are more prevalent than has been revealed by microscopy. This overlooked distribution should be considered by malaria control strategy makers. The genetic polymorphisms warrant further investigation.
\end{abstract}

\section{Background}

Malaria still continues to be a devastating global public health problem in more than 100 countries with 3.2 billion people being at risk [1]. Of this number, 300-500 million people contract the disease each year, resulting in 2-3 million deaths [2]. This includes 1 million children of less than five years of age [3].

The genus Plasmodium consists of nearly 200 species that infect humans, birds, reptiles and mammals. It belongs to the phylum Apicomplexa. Five Plasmodium species have been known to infect humans: $P$. falciparum, $P$. vivax, $P$. malariae, $P$. ovale, and $P$. knowlesi [4]. Much work on molecular phylogeny has focused on the relationship between Plasmodium species and the

\footnotetext{
* Correspondence: halkasemi@yahoo.com

'Department of Parasitology, Faculty of Medicine, University of Malaya,

50603 Kuala Lumpur, Malaysia

Full list of author information is available at the end of the article
}

origin of human malaria [5-7]. Although the $18 \mathrm{~s}$ rRNA gene appears to be robust for phylogenetic analysis, its sequences should be analysed carefully since the gene could be expressed as one of the three types during the different developmental stages of the malaria parasite $[4,8]$. Asexual type (A) and sporozoite type (S) were found in $P$. falciparum, $P$. berghei, $P$. vivax, and other species $[4,9]$ while oocyst type $(\mathrm{O})$ has been reported in P. vivax [10].

Yemen is a country in the East Mediterranean region where the highest incidence of malaria has been registered after Afghanistan [11] and is responsible for 4.9 deaths per 100000 per year and 43000 disability adjusted life years (DALYs) lost [12]. Giemsa microscopy is the most common diagnostic tool used in this country. This technique has drawbacks, however, in terms of detecting low parasitemic cases and the proper identification of mixed infection could actually underestimate the malaria
C Biomed Central

(c) 2010 Al-Mekhlafi et al; licensee BioMed Central Ltd. This is an Open Access article distributed under the terms of the Creative Commons Attribution License (http://creativecommons.org/licenses/by/2.0), which permits unrestricted use, distribution, and reproduction in any medium, provided the original work is properly cited. 
situation in Yemen. Thus, the molecular approach was applied for the first time, in this country, to investigate the molecular epidemiology of malaria. The study will indicate the magnitude of malaria co-infection which should be considered in clinical case management. In addition, malaria strains, genetic polymorphism and the molecular phylogeny of Yemen Plasmodium isolates will be studied.

\section{Methods}

\section{Study area}

The present study was conducted in five governorates with a total population of 7.9 million [13]. The selected governorates included Taiz and Hodeidah, which represent the mountainous hinterland and coastal areas, respectively; and Rymah, Dhamar and Sana'a from the highland areas. Most houses, especially in the rural areas, have a wooden roof. Occupations include agriculture, fishing, livestock and handicrafts. The peak time of malaria transmission in the coastal areas occurs in winter (October-April), while in the western mountains, the peak occurs in the summer (May-September). In the highland areas, which are located at more than 2000 metres above sea level, the transmission of malaria occurs throughout the year [14]. Anopheles arabiensis is the main vector in the country but Anopheles culicifacies plays an important role in the transmission of malaria in the coastal area. Anopheles sergenti has also been reported in the mountainous hinterland and highland areas [14]. There is a paucity of comparative data on mortality and morbidity caused by malaria in the three malaria endemic areas. An observational study showed that mortality rate among children with severe malaria was $2.4 \%$ and $3.5 \%$ for costal and hinterlands, respectively [15]. However, no data were available on mortality rate in the highland areas.

\section{Sample collection and genomic DNA preparation}

A total of 511 finger-prick blood samples were collected from symptomatic patients attending hospitals or medical centres for the treatment of malaria. Blood from finger pricks from febrile patients was collected and spotted on Whatman 3 MM filter papers (Whatman International Ltd., Maidstone, England) and slides to prepare thin and thick blood films. Each blood spot on Whatman filter paper was allowed to air-dry and was stored in a separate sealed plastic bag at room temperature until DNA extraction was required. Parasite genomic DNA was extracted from the blood collected on filter paper using QIAgen DNA Mini Kit blood and tissue (QIAGEN, cat. no. 51306, Germany) according to the manufacturer's instructions. Briefly, a disc was punched out from the blood spot using a pre-flamed paper puncher and placed in $1.5 \mathrm{ml}$ centrifuge tubes using clean, flamed forceps.

\section{Polymerase chain reaction (PCR)}

Genus-and species-specific nested PCR assays based on the $18 \mathrm{~s}$ rRNA gene were used to detect and identify Plasmodium species as previously reported [16]. Primary PCR was carried out using genus-specific primers (rPLU1 and rPLU5). PCR reaction was run in a total of $25 \mu \mathrm{L}$ reaction mixture containing $4 \mu \mathrm{L}$ genomic DNA, $1 \times \mathrm{i}$-Taq ${ }^{\mathrm{m}}$ buffer including $\mathrm{MgCl}_{2}$ (iNtRON BIOTECHNOLOGY, Seoul, Korea), $250 \mu \mathrm{M}$ dNTP (iNtRON BIOTECHNOLOGY, Seoul, Korea)), $200 \mathrm{nM}$ of each primer and $1.25 \mathrm{U}$ of $\mathrm{i}-\mathrm{Taq}^{\mathrm{im}} \mathrm{DNA}$ polymerase (iNtRON BIOTECHNOLOGY, Seoul, Korea). Secondary PCR was carried out using species-specific primers (rFAL1/ rFAL2, rVIV1/rVIV2, rMAL1/rMAL2 and rOVA1/ rOVA2); each primer pair was placed in a single tube. PCR mixtures were as mentioned above, except that $2 \mu \mathrm{L}$ of the first PCR product was used as a template in the secondary PCR.

The cycling conditions for primary PCR were as follows: an initial denaturation step at $95^{\circ} \mathrm{C}$ for 10 minutes, then 40 cycles at $94^{\circ} \mathrm{C}$ for 20 seconds, annealing at $55^{\circ} \mathrm{C}$ for 20 seconds, extension at $72^{\circ} \mathrm{C}$ for 1 minute, and a final extension at $72^{\circ} \mathrm{C}$ for 5 minutes. The reaction was terminated by reducing the product temperature to $10^{\circ}$ C. PCR products were stored at $-20^{\circ} \mathrm{C}$ until analysis. Secondary PCR used similar cycling conditions except that the number of cycles was reduced to 35 cycles.

\section{Cloning and DNA sequencing}

The PCR product comprising 17 positive samples (13 $P$. falciparum and $4 P$. vivax), representing different geographical areas, was selected for cloning and subsequent sequencing. A partial sequence $(\sim 1200 \mathrm{bp})$ of the $18 \mathrm{~s}$ rRNA gene was amplified with primers rPLU6 and rPLU5. PCR was carried out in a volume of $20 \mu \mathrm{l} \mathrm{mix-}$ ture containing $1 \times$ Phusion HF buffer (Finnzymes, Finland), $200 \mu \mathrm{M}$ of each dNTP (Finnzymes) and $400 \mathrm{nM}$ of each primer, and $1 \mathrm{U}$ of Phusion DNA Polymerase (Finnzymes). The PCR conditions were as follows: initial denaturation at $98^{\circ} \mathrm{C}$ for 30 seconds, followed by 40 cycles of amplification at $98^{\circ} \mathrm{C}$ for 7 seconds, $59^{\circ} \mathrm{C}$ for 20 seconds and $72^{\circ} \mathrm{C}$ for 48 seconds followed by a final extension step at $72{ }^{\circ} \mathrm{C}$ for 5 minutes. The amplified products were cloned into Zero Blunt ${ }^{\oplus}$ vector according to the manufacturer's instructions (cat. No. K2700-20; Invitrogen, USA). At least 20 colonies from each of transformation reactions were screened using the rPLU6 and rPLU5 primers. Amplification was done in a $20 \mu \mathrm{l}$ reaction mixture containing $1 \times$ reaction buffer $(5 \times$ Green Go Taq Flexi Buffer, Promega Madison USA), $2 \mathrm{mM} \mathrm{MgCl}$ (Promega), $200 \mathrm{mM}$ of each dNTP (Promega), $300 \mathrm{nM}$ of each primer and $0.5 \mathrm{U}$ Go Taq DNA polymerase (Promega,). PCR conditions were as follows: initial denaturation of $94^{\circ} \mathrm{C}$ for 10 minutes, followed by 
30 cycles of amplification at $94^{\circ} \mathrm{C}$ at 45 seconds, annealing at $55^{\circ} \mathrm{C}$ at 1 minute, extension at $72^{\circ} \mathrm{C}$ for 1 minute 30 seconds, followed by a final extension step at $72^{\circ} \mathrm{C}$ at 10 minutes. Plasmids from clones having the correct insert were extracted using QIAprep ${ }^{\oplus}$ Spin Miniprep kit (QIAgen, cat. no. 27106, Germany) following the manufacturer's instructions. Purified plasmid containing the insert was sequenced in both directions using the ABI PRISM $^{\odot}$ BigDye terminator v3.0 Ready Reaction Cycle Sequencing Kit (Applied Biosystems, USA) in an 3700 DNA Analyzer (Applied Biosystems, USA).

\section{Phylogenetic analysis}

DNA sequences (forward and reverse) were edited and the consensus sequence was created using the BioEdit. Consensus sequences were multiple-aligned with previously published sequences from the GenBank database using MEGA4 software http://www.megasoftware.net. Phylogenetic analysis was performed with the MEGA4 software. Two types of phylogenetic analysis were used on the aligned sequences to assess relationships among isolates; a distance-based Neighbor-Joining (NJ) analysis was performed calculated with the Kimura 2-parameter [17], and Maximum Parsimony (MP) analysis was performed using the Close-Neighbour-Interchange algorithm [18]. The reliability of the trees was assessed by the bootstrap method with 1,000 replications [19]. Similarity searches were carried out using the Basic Local Alignment Search Tool (BLAST) [20].

\section{Results}

A total of 511 blood samples from febrile patients were screened by using $18 \mathrm{~s}$ rRNA-based nested PCR. Of the 511 samples, 86 (16.8\%) were positive for malaria. A majority of the malarial infections were due to $P$. falciparum (80.3\%). Plasmodium vivax infections were seen in only $5.8 \%$ of the samples. Double infections with $P$. falciparum $+P$. vivax $(11.6 \%)$ and P. falciparum $+P$. malariae $(2.3 \%)$ were also detected (Table 1$)$. The PCR product of 13 P. falciparum isolates and four of $P$. vivax were selected from different geographical areas including the coastal area, the hinterland and the highland malaria endemic areas, and subjected to cloning and sequencing. The 17 sequences representing $P$. falciparum and $P$.

Table 1 Distribution of malaria species in the study population

\begin{tabular}{lc}
\hline Variable & Percentage \\
\hline Species & \\
P. falciparum & $69(80.3 \%)$ \\
P. vivax & $5(5.8 \%)$ \\
P. falciparum \&P. vivax & $10(11.6 \%)$ \\
P. falciparum \&P. malariae & $2(2.3 \%)$ \\
\hline
\end{tabular}

vivax from this study (Table 2) and 13 sequences representing human, bird and primate malaria from the GenBank database, were multiple aligned and analysed using the Neighbor-Joining (NJ) and Maximum Parsimony (MP) methods.

The NJ method (Figure 1) shows a main cluster for $P$. falciparum and $P$. reichenowi, the chimpanzee malaria (100 bootstrap). Within this main cluster, the Yemen $P$. facliparum isolates were placed into three sub-clusters. The first sub-cluster includes 8 isolates which grouped with $P$. falciparum 3D7 (GenBank Accession number AE014186) (100 bootstrap). The second subcluster comprises 4 Yemen isolates and P. falciparum Papua New Guinea (GenBank Accession number AF145334) (100 bootstrap). The Yemen P. falciparum isolate R73 diverged from other isolates and clustered with P. falciparum (GenBank Accession number AL844506) (99 bootstrap). The four Yemen isolates of $P$. vivax were grouped in one cluster with $P$. vivax Salvador1 (GenBank Accession number U03079) (100 bootstrap). The monkey malaria parasites (P. cynomolgi and $P$. knowlesi) were grouped with human $P$. vivax in one cluster (100 bootstrap). Bird malaria parasites $(P$. gallinaceum and $P$. lophurae) formed a separate cluster (100 bootstrap). The maximum parsimony tree (Additional file 1) is concordant in topology with the NJ tree.

The thirteen $18 \mathrm{~s}$ rRNA sequences of Yemen isolates of $P$. falciparum were multiple aligned against the 1135nucleotide sequence of 18 s rRNA of P. falciparum 3D7 (GenBank Accession number AE014186) to investigate the genetic diversity of $P$. falciparum in Yemen

Table 2 Yemen isolates of $P$. falciparum and $P$. vivax used in the phylogenetic analysis

\begin{tabular}{ccccc}
\hline SN & ID & Species & $\begin{array}{c}\text { Geographical } \\
\text { areas }\end{array}$ & $\begin{array}{c}\text { GenBank Accession } \\
\text { No. }\end{array}$ \\
\hline 3 & D122 & P. falciparum & Dhamar & HQ283210 \\
6 & D124 & P. falciparum & Dhamar & HQ283211 \\
11 & H12 & P. falciparum & Hodiedah & HQ283212 \\
2 & H61 & P. falciparum & Hodiedah & HQ283213 \\
13 & H87 & P. falciparum & Hodiedah & HQ283214 \\
10 & R73 & P. falciparum & Raymah & HQ283215 \\
9 & R117 & P. falciparum & Raymah & HQ283216 \\
5 & T71 & P. falciparum & Taiz & HQ283217 \\
7 & T91 & P. falciparum & Taiz & HQ283218 \\
4 & T113 & P. falciparum & Taiz & HQ283219 \\
1 & T116 & P. falciparum & Taiz & HQ283220 \\
8 & T122 & P. falciparum & Taiz & HQ283221 \\
12 & T124 & P. falciparum & Taiz & HQ283222 \\
17 & D24 & P. vivax & Dhamar & HQ283223 \\
14 & H15 & P. vivax & Hodiedah & HQ283224 \\
16 & H108 & P. vivax & Hodiedah & HQ283225 \\
15 & H120 & P. vivax & Hodiedah & HQ283226 \\
\hline
\end{tabular}




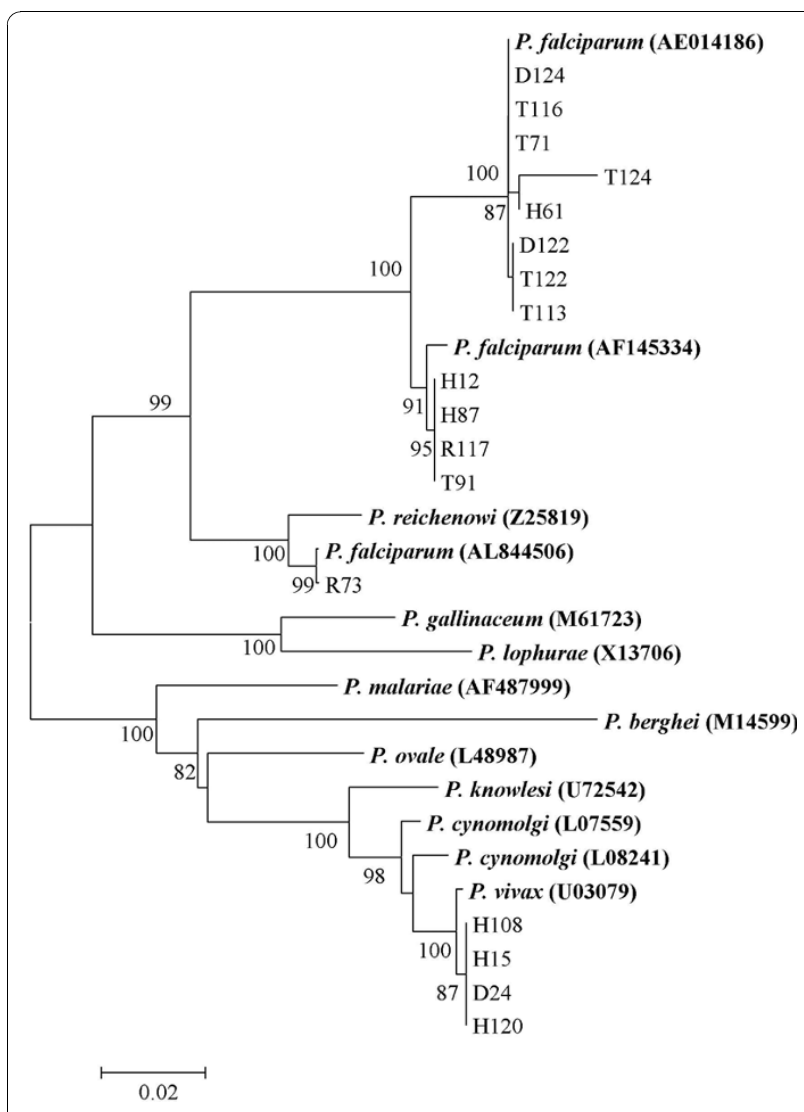

Figure 1 Neighbor-Joining (NJ) tree, constructed based on the nucleotide sequences of $18 \mathrm{~s}$ rRNA, displaying the relationships of 17 sequences representing $13 P$. Falciparum isolates and 4 sequences representing $4 P$. Vivax isolates Bootstrap support of more than $80 \%$ is indicated. Bold-type represents reference sequences for Plasmodium species from GenBank

(Additional file 2). The isolate T124 showed 25 SNP (19 substitutions, 1 insertion and 5 deletions). Isolates H61 and D122 showed 6 SNP (1 substitution and 5 deletions) and 8 SNP (1 substitution, 4 insertions and 3 deletions), respectively. Isolates $\mathrm{T} 122$ and T113 showed 7 SNP (1 substitution, 1 insertion and 5 deletions). Isolates D124, T116 and T71 had 10 SNP (10 deletions). Isolates R117, H12, T91 and H87, which were clustered together in the phylogenetic analysis, showed polymorphism in $5 \%$ of the 1135 -nucleatide sequence. The highest polymorphism (14.8\%) was noted in the isolate R73 which diverged in a separated cluster in the phylogenetic trees. The four sequences of the Yemen isolates of $P$. vivax were also aligned against 1058-nucleotide sequence of $P$. vivax Salvador1 (GenBank Accession number U03079). The isolate $\mathrm{H} 108$ had 7 SNP (6 substitutions and 1 insertion). The isolate $\mathrm{H} 15$ had 7 SNP (5 substitutions and 2 insertions). The isolates D24 and H124 showed 5 SNP (5 substitutions).
To determine whether the genetic diversity is due to the heterogeneous types of Plasmodium $18 \mathrm{~s}$ rRNA or not, phylogenetic trees were re-constructed based on our sequences and sequences representing different types of Plasmodium 18 s rRNA (A, O or S type) from the GenBank database. Both the NJ tree (Figure 2) and MP tree (Additional file 3) were consistent, placing all the Yemen isolates of P. falciparum except isolate R73 into one cluster and clade with P. falciparum type-S (GenBank Accession number M19173) (100 bootstrap). The isolate R73 was much closer to P. falciparum $18 \mathrm{~s}$ rRNA type-A (GenBank Accession number M19173) (100 bootstrap). All P. vivax isolates were grouped with $P$. vivax type-A in one cluster (GenBank Accession number U07367) (100 bootstrap).

\section{Discussion}

This is the first study to apply molecular techniques to study the epidemiology and phylogeny of malaria in Yemen. Malaria infection rate, using $18 \mathrm{~s}$ rRNA-based nested PCR, was $16.8 \%$ which is comparable with previous studies carried out in Yemen based on Giemsa microscopy [15,21-25]. PCR results showed that approximately $12 \%$ of the positive malaria cases were mixed infection of $P$. falciparum and $P$. vivax or $P$. malariae which is a higher rate than has been hitherto reported. The high proportion of

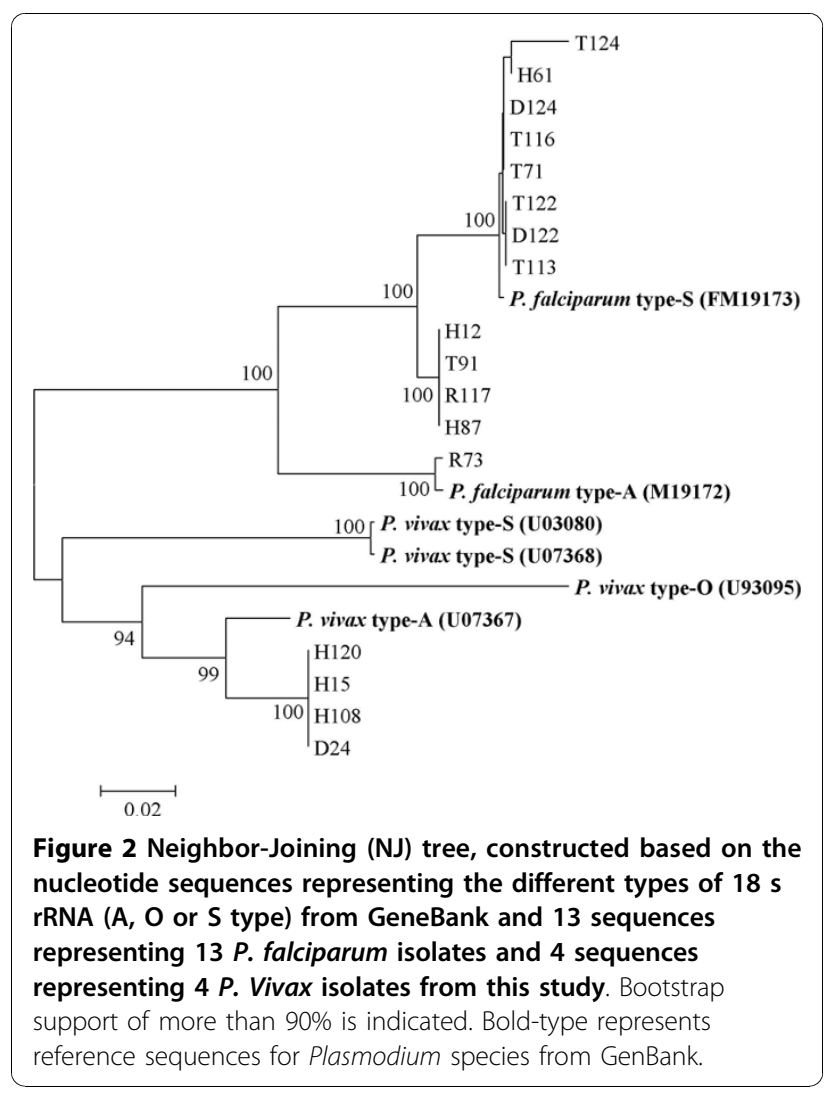


mixed infection using molecular identification could be explained by the high sensitivity of PCR [26] and the low level parasitaemia of the dominated species which is under the diagnostic threshold of Giemsa microscopy. Accurate identification of mixed infection with malaria species is very important for proper clinical case management. Malaria treatment policy differs depending on the infecting species. Thus, in the case of mixed infection, if only one species is treated, the other may establish a new episode of malaria.

Plasmodium falciparum is the dominant malaria species in Yemen followed by $P$. vivax, whereas $P$. malariae has rarely been reported in Yemen. The current study highlighted the possibility that $P$. malariae is distributed more than expected and it is often overlooked due to low levels of parasitaemia and the shortcoming of microscopy, the only technique used for malaria detection and identification in the country. Furthermore, $P$. malariae may be obscured by the dominant species. The underestimation of $P$. malariae distribution has been reported in other countries [27-30]

Phylogenetic analysis using the NJ method grouped Yemen isolates of $P$. falciparum into one main cluster. Within the main cluster, the Yemen isolates were placed in three sub-clusters (Figure 1). These clusters were supported by cladograms using the MP method. One subcluster contained 8 isolates and P. falciparum 3D7 (GenBank Accession number AE014186) [31]. The second subcluster included four isolates and P. falciparum (GenBank Accession number AF145334) which was isolated from Papua New Guinea [32]. The isolate R73 formed a separate cluster with P. falciparum (GenBank Accession number AL844506). Phylogenetic analysis based on our sequences and sequences from the GenBank representing the secondary structure of $18 \mathrm{~s}$ rRNA showed that isolate R73 that diverged in a separate cluster may be A-type ribosomal rRNA, while the other two sub-clusters are Stype rRNA. Thus, genetic diversity within the Yemen isolates that grouped in one cluster with the S-type rRNA may be not due to the heterogeneity of $18 \mathrm{~s}$ rRNA genes. Genetic diversity may provide a mechanism for drug resistance which may affect any intervention strategy based on treatment. Furthermore, PCR-based specific diagnosis of Plasmodium species may fail due to sequence variations in the priming regions, thus leading to false negative results. The low sensitivity of PCR-based diagnosis due to genetic mutations has been previously reported [30].

\section{Conclusions}

In conclusion, malaria is a major public health problem in Yemen. Although P. falciparum is predominant, $P$. vivax, $P$. malariae and mixed infections are more prevalent than has been revealed by microscopy. This overlooked distribution should be considered by malaria control strategy makers. Sequences from P. falciparum isolates showed high genetic polymorphisms that may not be related to the variants of ribosomal RNA expressed in the different stages of malaria parasites which warrant further studies.

\section{Additional material}

\begin{abstract}
Additional file 1: Maximum Parsimony (MP) tree. The tree was constructed based on the nucleotide sequences of $18 \mathrm{~s}$ rRNA, displaying the relationship of 17 sequences representing $13 P$. falciparum isolates and 4 sequences representing $4 P$. vivax isolates. Bootstrap support of more than $60 \%$ is indicated. Bold-type represents reference sequences for Plasmodium species from GenBank.

Additional file 2: Multiple alignment of partial $18 \mathrm{~s}$ rRNA sequences. Multiple alignment of partial $18 \mathrm{~s}$ rRNA sequences of Yemen Plasmodium falciparum isolates against $P$. falciparum 3D7 (GenBank accession numbers AE014186). Dots represent sequence identities. Dashes denote no nucleotides.

Additional file 3: Maximum parsimony (MP) tree. The tree was constructed based on nucleotide sequences representing the different types of $18 \mathrm{~s}$ rRNA (A, O or S type) from GeneBank and 13 sequences representing $13 P$. falciparum isolates and 4 sequences representing $4 P$. vivax isolates from this study. Bootstrap support of more than $90 \%$ is indicated. Bold-type represents reference sequences for Plasmodium species from GenBank.
\end{abstract}

\section{Acknowledgements}

The authors thank all the technical staff in the hospitals and medical centres in the five governorates, especially Sultan Ayesh, Fawaz Al-Soroorey, Ashraf Saleh and Ahlam Al-Kobati. Thanks are also due to Entesar Mansour M.H and Nemah O. M. Bin Shuaib for their assistance in the laboratory work. The authors also thank Dr. Hesham M. Al-Mekhlafi for his assistance. The study was funded by a research grant from the University of Malaya, Kuala Lumpur, Malaysia (Research Code PS175/2008C).

\section{Author details}

${ }^{1}$ Department of Parasitology, Faculty of Medicine, University of Malaya, 50603 Kuala Lumpur, Malaysia. ${ }^{2}$ Department of Parasitology, Faculty of Medicine and Health Sciences, Sana'a University, Sana'a - Yemen.

\section{Authors' contributions}

AMQA, MAKM, FMY and AAA and designed the study; AMQA dealt with study subjects in the field, carried out the laboratory work and collected the data; AMQA and MAKM performed the statistical analysis; AMQA, MAKM, FMY and AAA and interpreted the data; AMQA and MAKM drafted the manuscript; AMQA, FMY, AAA, MAKM contributed to the revision of the manuscript. All authors read and approved the final manuscript. AMQA and FMY are guarantors of the paper.

\section{Competing interests}

The authors declare that they have no competing interests.

Received: 8 October 2010 Accepted: 19 November 2010

Published: 19 November 2010

\section{References}

1. WHO: World Malaria Report. World Health Organization; 2005.

2. Snow RW, Guerra CA, Noor AM, Myint HY, Hay SI: The global distribution of clinical episodes of Plasmodium falciparum malaria. Nature 2005, 434:214-217.

3. Sachs J, Malaney P: The economic and social burden of malaria. Nature 2002, 415:680-685.

4. Nishimoto Y, Arisue N, Kawai S, Escalante AA, Horii T, Tanabe K, Hashimoto T: Evolution and phylogeny of the heterogeneous cytosolic 
SSU rRNA genes in the genus Plasmodium. Mol Phylogenet Evol 2008, 47:45-53.

5. Escalante AA, Ayala FJ: Evolutionary origin of Plasmodium and other Apicomplexa based on rRNA genes. Proc Natl Acad Sci USA 1995, 92:5793-5797.

6. Escalante AA, Cornejo OE, Freeland DE, Poe AC, Durrego E, Collins WE, Lal AA: A monkey's tale: the origin of Plasmodium vivax as a human malaria parasite. Proc Natl Acad Sci USA 2005, 102:1980-1985.

7. Waters AP, Higgins DG, McCutchan TF: Plasmodium falciparum appears to have arisen as a result of lateral transfer between avian and human hosts. Proc Natl Acad Sci USA 1991, 88:3140-3144.

8. Escalante AA, Goldman IF, De Rijk P, De Wachter R, Collins WE, Qari SH, Lal AA: Phylogenetic study of the genus Plasmodium based on the secondary structure-based alignment of the small subunit ribosomal RNA. Mol Biochem Parasitol 1997, 90:317-321.

9. van Spaendonk RM, Ramesar J, van Wigcheren A, Eling W, Beetsma AL, van Gemert GJ, Hooghof J, Janse CJ, Waters AP: Functional equivalence of structurally distinct ribosomes in the malaria parasite, Plasmodium berghei. J Biol Chem 2001, 276:22638-22647.

10. Li J, Gutell RR, Damberger SH, Wirtz RA, Kissinger JC, Rogers MJ, Sattabongkot J, McCutchan TF: Regulation and trafficking of three distinct $18 \mathrm{~S}$ ribosomal RNAs during development of the malaria parasite. $\mathrm{J} \mathrm{Mol}$ Biol 1997, 269:203-213.

11. WHO: World Malaria Report. World Health Organization; 2009.

12. WHO: The global burden of disease: 2004 update Geneva: World Health Organization; 2008.

13. Ministry of planning and international cooperation Y: National census of Yemen. 2004.

14. NMCP: Malaria Report. Yemen: National Malaria Control Programme; 2002.

15. Al-Taiar A, Jaffar S, Assabri A, Al-Habori M, Azazy A, Al-Mahdi N, Ameen K, Greenwood BM, Whitty CJ: Severe malaria in children in Yemen: two site observational study. BMJ 2006, 333:827.

16. Singh B, Bobogare A, Cox-Singh J, Snounou G, Abdullah MS, Rahman HA: A genus- and species-specific nested polymerase chain reaction malaria detection assay for epidemiologic studies. Am J Trop Med Hyg 1999, 60:687-692.

17. Kimura M: A simple method for estimating evolutionary rates of base substitutions through comparative studies of nucleotide sequences. $J$ Mol Evol 1980, 16:111-120.

18. Nei M, Kumar S: Molecular evolution and phylogenetics. New York: Oxford University Press; 2000.

19. Felsenstein J: Confidence limits on phylogenies: an approach using the bootstrap. Evolution 1985, 39:9.

20. Altschul SF, Madden TL, Schaffer AA, Zhang J, Zhang Z, Miller W, Lipman DJ: Gapped BLAST and PSI-BLAST: a new generation of protein database search programs. Nucleic Acids Res 1997, 25:3389-3402.

21. Ahmed SM, Abd Al-Rhim SK, Mohamedani AA, Habour AB, Sadek AA Malaria parasitemia during delivery. Saudi Med J 2002, 23:684-688.

22. Alkadi HO, Al-Maktari MT, Nooman MA: Chloroquine-resistant Plasmodium falciparum local strain in Taiz Governorate, Republic of Yemen. Chemotherapy 2006, 52:166-170.

23. Al-Maktari MT, Bassiouny HK: Malaria status in Al-Hodeidah Governorate, Republic of Yemen. Part II: Human factors causing the persistence of chloroquine resistant P. falciparum local strain. J Egypt Soc Parasitol 2003, 33:829-839.

24. Al-Taiar A, Jaffar S, Assabri A, Al-Habori M, Azazy A, Al-Gabri A, Al-Ganadi M, Attal $B$, Whitty CJ: Who develops severe malaria? Impact of access to healthcare, socio-economic and environmental factors on children in Yemen: a case-control study. Trop Med Int Health 2008, 13:762-770.

25. Bin Mohanna MA, Bin Ghouth AS, Rajaa YA: Malaria signs and infection rate among asymptomatic schoolchildren in Hajr Valley, Yemen. East Mediterr Health J 2007, 13:35-40.

26. Muller O, Ye M, Louis VR, Sie A: Malaria in sub-Saharan Africa. Lancet 2009, 373:122.

27. Zhou M, Liu Q, Wongsrichanalai C, Suwonkerd W, Panart K, Prajakwong S, Pensiri A, Kimura M, Matsuoka H, Ferreira MU, Isomura S, Kawamoto F: High prevalence of Plasmodium malariae and Plasmodium ovale in malaria patients along the Thai-Myanmar border, as revealed by acridine orange staining and PCR-based diagnoses. Trop Med Int Health 1998, 3:304-312.

28. Paxton LA, Slutsker L, Schultz LJ, Luby SP, Meriwether R, Matson $P$, Sulzer AJ: Imported malaria in Montagnard refugees settling in North
Carolina: implications for prevention and control. Am J Trop Med Hyg 1996, 54:54-57.

29. Snounou G, Pinheiro L, Goncalves A, Fonseca L, Dias F, Brown KN, do Rosario VE: The importance of sensitive detection of malaria parasites in the human and insect hosts in epidemiological studies, as shown by the analysis of field samples from Guinea Bissau. Trans R Soc Trop Med Hyg 1993, 87:649-653.

30. Liu Q, Zhu S, Mizuno S, Kimura M, Liu P, Isomura S, Wang X, Kawamoto F: Sequence variation in the small-subunit rRNA gene of Plasmodium malariae and prevalence of isolates with the variant sequence in Sichuan, China. J Clin Microbiol 1998, 36:3378-3381.

31. Gardner MJ, Hall N, Fung E, White O, Berriman M, Hyman RW, Carlton JM, Pain A, Nelson KE, Bowman S, et al: Genome sequence of the human malaria parasite Plasmodium falciparum. Nature 2002, 419:498-511.

32. Mehlotra RK, Lorry K, Kastens W, Miller SM, Alpers MP, Bockarie M, Kazura JW, Zimmerman PA: Random distribution of mixed species malaria infections in Papua New Guinea. Am J Trop Med Hyg 2000, 62:225-231.

doi:10.1186/1756-3305-3-110

Cite this article as: Al-Mekhlafi et al:: Molecular epidemiology of Plasmodium species prevalent in Yemen based on $18 \mathrm{~s}$ rRNA. Parasites \& Vectors 2010 3:110.

\section{Submit your next manuscript to BioMed Central and take full advantage of:}

- Convenient online submission

- Thorough peer review

- No space constraints or color figure charges

- Immediate publication on acceptance

- Inclusion in PubMed, CAS, Scopus and Google Scholar

- Research which is freely available for redistribution 